\title{
Sudden hearing loss after a methadone overdose
}

\author{
F. A. van Gaalen · E. A. Compier · A. J. Fogteloo
}

Received: 1 August 2008 / Accepted: 18 February 2009 / Published online: 5 March 2009

(c) The Author(s) 2009. This article is published with open access at Springerlink.com

Sir,

We read with interest the case report by Schrock et al. "Sudden sensorineural hearing loss after heroin injection" published in the March 2008 issue of this journal [1].

Over the years, several cases of bilateral sudden sensorineural hearing loss (SHL) after a heroin overdose have been described, including the recent case in this journal. Although these cases all suggest that a large dose of opiates may cause acute hearing loss, the fact that the heroin used in these cases was illegal makes it impossible to rule out an effect of a heroin contaminant. We now report a patient who, after taking an accidental overdose of prescribed methadone, developed acute, reversible bilateral hearing loss.

After taking 15 tablets of $5 \mathrm{mg}$ of methadone a 37-yearold man was admitted to our hospital with nausea, deafness, confusion, and a low respiratory rate. His condition responded well to a single dose of naloxone and a urine sample tested positive for methadone. After regaining full consciousness the patient complained of profound hearing loss with a mild tinnitus. He had no vestibular symptoms or aural fullness.

Neurological examination was normal and examination of the tympanic membranes showed no abnormalities. The patient had no history of hearing problems, had never used

F. A. van Gaalen $(\bowtie) \cdot$ E. A. Compier · A. J. Fogteloo

Department of General Internal Medicine,

C4-R, Leiden University Medical Centre,

PO Box 9600, 2300 RC Leiden, The Netherlands

e-mail: f.a.van_gaalen@lumc.nl opiates before and admitted that he had taken the methadone from his brother who was enrolled in a heroin withdrawal program.

An ototoxic medication effect was suspected but the patient consistently denied the use of other drugs or medications including over-the-counter analgesics in the previous weeks. Pure tone audiometry performed a day later revealed bilateral SHL of on average $-57 \mathrm{db}$ in the right ear and $-40 \mathrm{~dB}$ in the left ear (Fig. 1). Within a day, the patient reported marked improvement in hearing and a repeated audiometry 10 days later was completely normal.

In our case of sudden, bilateral SHL after an overdose of prescribed methadone an effect of a contaminant is very unlikely. As our case shows that a methadone overdose can precede bilateral SHL, this supports the claim that in the previous cases of heroin induced hearing loss, heroin itself was indeed the cause. A possible explanation for the fact that this is only the first reported case of bilateral SHL after a methadone overdose is that methadone overdoses are much rarer than heroin overdoses [2].

In theory, it is possible that in our case (and the other cases) the hearing loss was purely coincidental and not because of the overdose itself. Nevertheless, it should be kept in mind that SHL and in particular when it is bilateral is rare and that there was a clear time relation between the overdose and the onset of the hearing loss.

In conclusion our case shows that, as with heroin, a methadone overdose may herald sudden bilateral SHL.

Open Access This article is distributed under the terms of the Creative Commons Attribution Noncommercial License which permits any noncommercial use, distribution, and reproduction in any medium, provided the original author(s) and source are credited. 

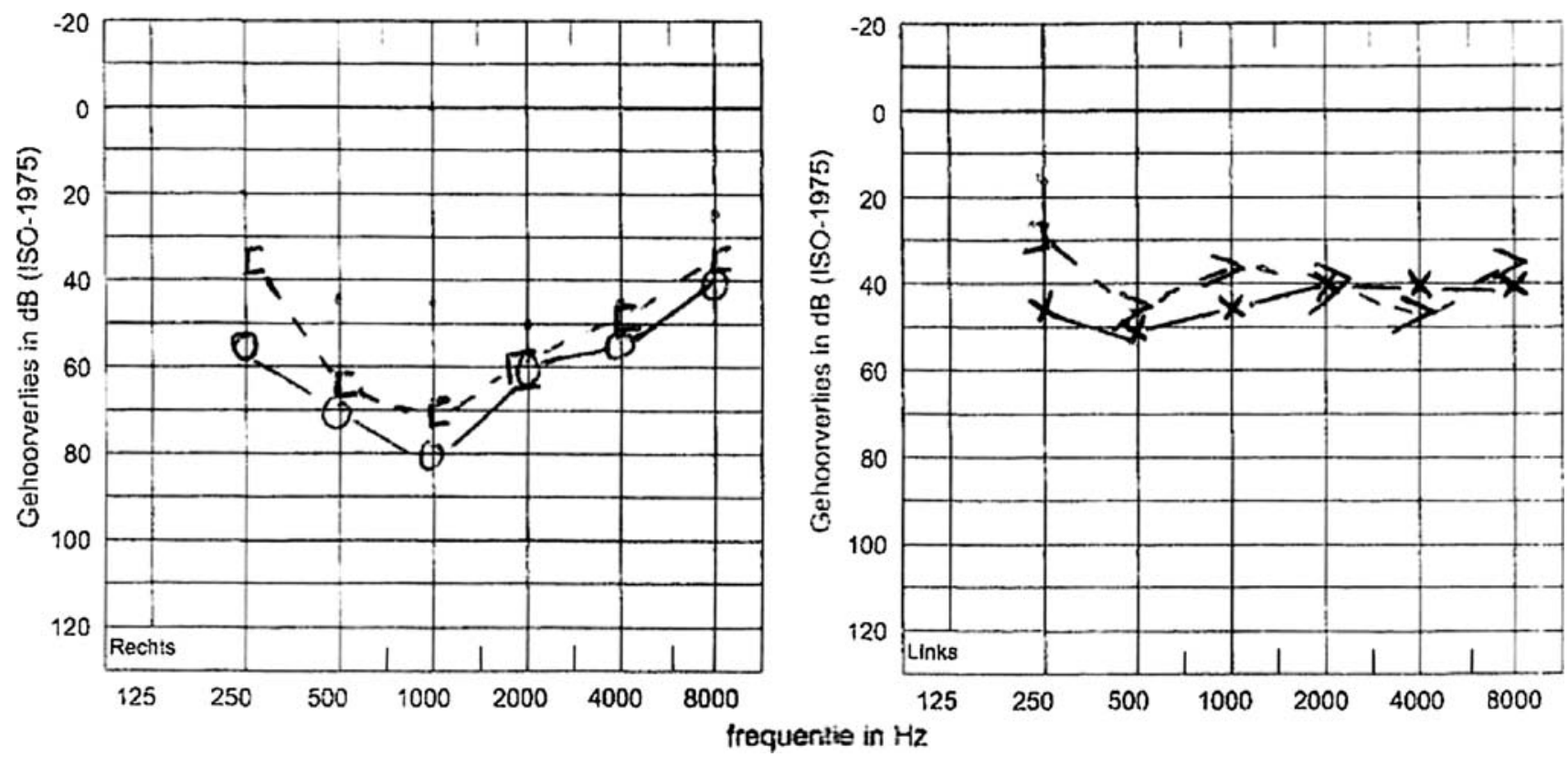

Fig. 1 Audiogram recorded a day after a methadone overdose. The audiograms is set out with frequency in hertz $(\mathrm{Hz})$ on the horizontal axis (logarithmic scale), and a linear hearing level scale (in decibel

$(\mathrm{dB})$ on the vertical axis. Right ear (left) and left ear (right). Symbols: "O" and "X" are air conduction and "[" and "]"”" indicate bone conduction

\section{References}

1. Schrock A, Jakob M, Wirz S, Bootz F (2008) Sudden sensorineural hearing loss after heroin injection. Eur Arch Otorhinolaryngol 265(5):603-606. doi:10.1007/s00405-007-0495-6

2. Bryant WK, Galea S, Tracy M, Markham PT, Tardiff KJ, Vlahov D (2004) Overdose deaths attributed to methadone and heroin in New York City, 1990-1998. Addiction 99(7):846-854. doi:10.1111/ j.1360-0443.2004.00693.x 